\title{
MOLECULAR ECOLOGY
}

\section{Speciation processes in putative island endemic sister bat species: false impressions from mitochondrial DNA and microsatellite data}

\begin{tabular}{|c|c|}
\hline Journal: & Molecular Ecology \\
\hline Manuscript ID & MEC-15-0787.R1 \\
\hline Manuscript Type: & Original Article \\
\hline Date Submitted by the Author: & $\mathrm{n} / \mathrm{a}$ \\
\hline Complete List of Authors: & $\begin{array}{l}\text { Kuo, Hao-Chih; Queen Mary, University of London, School of Biological and } \\
\text { Chemical Sciences } \\
\text { Chen, Shiang-Fan; National Taipei University, Center for General Education } \\
\text { Fang, Yin-Ping; National Chiayi University, Department of Biological } \\
\text { Resources } \\
\text { Cotton, James; Queen Mary, University of London, School of Biological and } \\
\text { Chemical Sciences } \\
\text { Parker, Joe; Queen Mary, University of London, School of Biological and } \\
\text { Chemical Sciences } \\
\text { Csorba, Gábor; Hungarian Natural History Museum, Department of Zoology } \\
\text { Lim, Burton; Royal Ontario Museum, } \\
\text { Eger, Judith; Royal Ontario Museum, Natural History; } \\
\text { Chen, Chia-Hong; Shei-Pa National Park Headquarters, } \\
\text { Chou, Cheng-Han; Endemic Species Research Institute, Division of Zoology } \\
\text { Rossiter, Stephen; Queen Mary, University of London, School of Biological } \\
\text { and Chemical Sciences; }\end{array}$ \\
\hline Keywords: & $\begin{array}{l}\text { Non-allopatric divergence, introgressive hybridization, gene flow, Taiwan, } \\
\text { Murina }\end{array}$ \\
\hline
\end{tabular}

\section{SCHOLARONE ${ }^{m}$


1 Speciation processes in putative island endemic sister bat species: false

2 impressions from mitochondrial DNA and microsatellite data

4 HAO-CHIH KUO, ${ }^{1}$ SHIANG-FAN CHEN, ${ }^{2}$ YIN-PING FANG,${ }^{3}$ JAMES A COTTON, $^{1} * *$ JOE 5 D PARKER, ${ }^{1}$ GÁBOR CSORBA, ${ }^{4}$ BURTON K LIM, ${ }^{5}$ JUDITH L EGER, ${ }^{5}$ CHIA-HONG

$6 \mathrm{CHEN}^{6}{ }^{6} \mathrm{CHENG}-\mathrm{HAN} \mathrm{CHOU}^{7}$ and STEPHEN J ROSSITER ${ }^{1 *}$

$7 \quad{ }^{1}$ School of Biological and Chemical Sciences, Queen Mary University of London, London E1

8 4NS, UK; ${ }^{2}$ Center for General Education, National Taipei University, New Taipei City 23741,

9 Taiwan; ${ }^{3}$ Department of Biological Resources, National Chiayi University, Chiayi City 60004,

10 Taiwan; ${ }^{4}$ Department of Zoology, Hungarian Natural History Museum, 1088 Budapest, Hungary;

$11{ }^{5}$ Department of Natural History, Royal Ontario Museum, Toronto, ON M5S 2C6, Canada; ${ }^{6}$ Shei-

12 Pa National Park Headquarters, Miaoli County 36443, Taiwan; ${ }^{7}$ Division of Zoology, Endemic

13 Species Research Institute, Nantou County 552, Taiwan

15 Keywords: Non-allopatric divergence, introgressive hybridization, gene flow, Taiwan, Murina

17 *Correspondence: Stephen Rossiter

18 Fax: +44 (0) 207882 7732;

19 E-mail: s.j.rossiter@qmul.ac.uk

$21 * *$ Current address: Wellcome Trust Sanger Institute, Wellcome Trust Genome Campus,

22 Cambridgeshire CB10 1SA, UK 
23 Abstract

24 Cases of geographically restricted co-occurring sister taxa are rare and may point to potential 25 divergence with gene flow. The two bat species Murina gracilis and M. recondita are both 26 endemic to Taiwan and are putative sister species. To test for non-allopatric divergence and gene 27 flow in these taxa, we generated sequences using Sanger and Next Generation Sequencing, and 28 combined these with microsatellite data for coalescent-based analyses. MtDNA phylogenies 29 supported the reciprocally monophyletic sister relationship between M. gracilis and M. recondita, 30 however, clustering of microsatellite genotypes revealed several cases of species admixture 31 suggesting possible introgression. Sequencing of microsatellite flanking regions revealed that 32 admixture signatures stemmed from microsatellite allele homoplasy rather than recent 33 introgressive hybridization, and also uncovered an unexpected sister relationship between $M$. 34 recondita and the continental species $M$. eleryi, to the exclusion of $M$. gracilis. To dissect the 35 basis of these conflicts between ncDNA and mtDNA, we analysed sequences from 10 36 anonymous ncDNA loci with *BEAST and isolation-with-migration (IM) and found two distinct 37 clades of $M$. eleryi, one of which was sister to $M$. recondita. We conclude that Taiwan was 38 colonized by the ancestor of $M$. gracilis first, followed by the ancestor of $M$. recondita after a 39 period of allopatric divergence. After colonization, the mitochondrial genome of M. recondita 40 was replaced by that of the resident $M$. gracilis. This study illustrates how apparent signatures of 41 sympatric divergence can arise from complex histories of allopatric divergence, colonization and 42 hybridization, thus highlighting the need for rigorous analyses to distinguish between such 43 scenarios. 


\section{Introduction}

The expectation that drift and selection will more easily establish genetic differentiation

47 between populations with little or no gene flow has led to the overwhelming view that most cases

48 of speciation must involve a period of geographical isolation (e.g. Barraclough \& Vogler 2000;

49 Turelli et al. 2001). Despite this, theoretical models have proposed routes by which parapatric

50 and sympatric speciation (hereafter collectively referred to as 'non-allopatric speciation') can

51 proceed (Slatkin 1982; Rice 1984; Dieckmann \& Doebeli 1999; Gavrilets \& Waxman 2002) and

52 these studies have been complemented by several empirical examples that suggest non-allopatric

53 speciation could be more common in nature than was previously assumed (reviewed in Via 2001).

In evaluating potential cases of non-allopatric speciation, several researchers have

56 advocated using biogeographical information. Lynch (1989) proposed that range overlap and

57 relative range sizes of sister species or supra-specific taxa could be used to infer geographical

58 modes of speciation, with substantial overlap pointing to sympatric speciation, and little overlap

59 indicating allopatric speciation. Such reasoning has been used to argue for greater frequencies of

60 sympatric speciation in nature than previously thought (e.g. Mattern \& McLennan 2000), but has

61 also received criticism given that a key assumption - that geographical ranges of natural

62 organisms are constant through time - is probably seldom true (reviewed in Losos \& Glor 2003;

63 Coyne 2007).

Extending the logic of studying range overlap, Coyne and Price (2000) emphasized the

66 value of looking for 'sister species' (species that are each other's closest relatives) that are both 
67 vagile yet restricted geographically to the same small area, such as an oceanic island (also see

68 White 1978). Under these conditions, they suggested that sympatric rather than allopatric

69 divergence is a likely explanation for such overlapping ranges. Coyne and Price (2000) searched

70 for oceanic islands (area 0.8 to $3500 \mathrm{~km}^{2}$ ) on which at least one endemic bird species occurred.

71 By assessing whether or not each taxon's sister species co-existed on the same island or

72 archipelago, consistent with in situ divergence, they found no clear evidence supporting non-

73 allopatric speciation in the species pairs studied. Kisel and Barraclough (2010) extended this

74 approach to diverse taxa and found that potential cases of in situ speciation in highly mobile

75 animals such as moths and mammals were associated with larger island areas $\left(140,000 \mathrm{~km}^{2}\right.$ and

$76420,000 \mathrm{~km}^{2}$, respectively), while smaller areas were associated with possible examples from

77 plants and less mobile animals (snails and lizards). Thus there appears little evidence for

78 sympatric speciation when applying Coyne and Price's (2000) criteria for island-dwelling taxa, at

79 least for highly mobile animals.

A common criticism of focusing on geography in speciation biology is that it detracts

82 from the underlying gene dynamics (see Hey 2006; Fitzpatrick et al. 2009). Indeed it is implicitly

83 assumed, but rarely tested, that non-allopatric divergence typically occurs in the face of gene

84 flow, especially where reproductive isolation is thought to arise via ecological shifts (e.g.

85 Kingston \& Rossiter 2004; Jackson 2008; Forbes et al. 2009) rather than by post-zygotic

86 mechanisms (e.g. Husband \& Sabara 2004). Recently several empirical studies have applied

87 newly developed isolation-with-migration (IM) models (Hey \& Nielsen 2004; Hey 2010b) to

88 demonstrate historical gene flow among closely related taxa, attributable to either non-allopatric 
89 divergence (e.g. Hey 2006; Nadachowska \& Babik 2009) or, alternatively, allopatric divergence

90 followed by secondary contact (e.g. Llopart et al. 2002; Geraldes et al. 2006). Generally,

91 however, most such studies have been unable to date the occurrence of gene flow; simulations

92 show that IM methods cannot provide reliable estimates of the timing of historical gene flow

93 (Strasburg \& Rieseberg 2011). Consequently, to distinguish between cases of taxa that have

94 formed sympatrically with gene flow versus those that evolved while geographically isolated but

95 which have subsequently undergone secondary gene flow, additional information can be

96 informative, including knowledge of the broader patterns of genetic affiliations with other

97 populations and taxa, and the potential for historical range shifts in light of climatic fluctuations.

While interpretations of speciation based on either range overlap or isolation-withmigration each have their respective shortcomings, these approaches are complementary and

101 together might offer a more powerful means of testing for non-allopatric divergence. Here we

102 combine these methods to study the origin of two newly discovered bat species Murina gracilis

103 and $M$. recondita on Taiwan (Kuo et al. 2009), which phylogenetic reconstructions using

104 mitochondrial DNA (mtDNA) indicate are sister taxa (Kuo 2004, 2013). Such cases of 105 geographically restricted co-occurring sister species are exceptionally rare in bats; in their review,

106 Kisel and Barraclough (2010) identified two congeneric bat species on New Zealand as the best 107 potential example. Given that Taiwan is a much smaller island $\left(36,000 \mathrm{~km}^{2}\right)$ with respect to the 108 expected mobility of flying mammals, M. gracilis and M. recondita meet Coyne and Price's 109 (2000) criteria and thus offer an excellent opportunity to test for non-allopatric divergence.

110 Moreover, the fact that these taxa show contrasting altitudinal preferences, with the former 
111 tending to occur at higher elevations (Kuo 2004, 2013), further suggests the potential for 112 ecological speciation linked to niche differentiation. We generated datasets of nuclear and 113 mitochondrial markers for both species and applied IM-based modeling approaches alongside 114 other population genetic analyses for gene flow at different scales. Since a detailed dissection of 115 the divergence process requires a thorough understanding of the relationship between the two 116 focal taxa, we also generated and analysed sequences for the continental congeneric (and also 117 recently described) species $M$. eleryi (Furey et al. 2009). We hypothesized that given their close 118 relationship and endemic status on Taiwan, M. gracilis and M. recondita likely diverged in situ 119 on this island and, therefore, would show evidence of a sister relationship across all markers, as 120 well as evidence of divergence in the face of gene flow. 


\section{$121 \quad$ Materials and methods}

122 We focused on three newly described species of tube-nosed bat from East Asia. Murina gracilis 123 and M. recondita are considered endemic to Taiwan (Kuo et al. 2009), while M. eleryi has been 124 recorded in Southern China, Vietnam and Laos (Furey et al. 2009; Eger \& Lim 2011). Sibling 125 relationships among these three taxa have been inferred from both morphometric comparisons 126 and mtDNA phylogenetic reconstructions, with the latter unambiguously supporting a 127 relationship of ((M. gracilis, M. recondita), M. eleryi) (see Systematic notes on the focal species, 128 Supporting information). Although little is known about their respective ecologies, M. gracilis 129 occurs at elevations of $>1500$ metres above-sea-level (asl), whereas $M$. recondita and M. eleryi

130 both occur at lower elevations of $<1500$ metres asl (Kuo et al. 2014; Table S2, Supporting 131 information).

133 Assessment of contemporary gene flow between M. gracilis and M. recondita

134 To test for contemporary gene flow between $M$. gracilis and $M$. recondita we screened 135 populations for evidence of genetic admixture using the Bayesian clustering method implemented 136 in STRUCTURE 2.3.2 (Pritchard et al. 2000). Genotypes of 106 M. gracilis and 144 M. 137 recondita at 14 microsatellite loci (A4, A9, A10, A104, A109, A118, A122, B5, B9, B114, B121, 138 B124, D110, and D117; see Kuo et al. 2013) were examined under a model that assumed 139 independent allele frequencies among genetic clusters and allowed for mixed ancestries among 140 individuals. We ran ten replicates of Markov chain Monte Carlo (MCMC) for between two and 141 ten clusters $(\mathrm{K})$ with each MCMC comprising 0.75 million iterations for sampling, and the same 142 number for burn-in. To assess consistency across replicates we used the CLUMPP procedure 
143 (Jakobsson \& Rosenberg 2007) and visualized the resulting plots using DISTRUCT (Rosenberg 144 2004). Plots under the $\mathrm{K}$ value that best fitted the data, as justified according to the rationale 145 proposed by Pritchard et al. (2000), were inspected for signatures of genetic admixture.

For each individual bat we estimated the proportion of its genetic composition, as 148 measured by the ancestry coefficient q (Pritchard et al. 2000), that could be assigned to its own 149 species, summing across multiple clusters where relevant. A few individuals of $M$. recondita 150 showed $<0.9$ assignment to their own species (see Results), potentially reflecting genetic 151 introgression from $M$. gracilis. Alternatively, such signatures might arise through allele size 152 homoplasy ( $\mathrm{SH}$ ), which can occur in microsatellites due to their hypervariable nature (Estoup et 153 al. 2002). To identify those loci driving signatures of admixture, we repeated our STRUCTURE 154 and CLUMPP analyses under the best-fitting $\mathrm{K}$ but excluded each locus one-by-one. Three 155 replicate runs were conducted for each pruning scheme and, for each bat showing mixed ancestry, 156 we recalculated $\mathrm{q}$ assigned into its own species under each pruning scheme. Loci that when 157 pruned led to an increase in q (compared with values based on the full dataset) were regarded as 158 likely candidates for introgression or SH. To distinguish between these scenarios, we amplified 159 and sequenced the corresponding flanking regions of those loci contributing most to admixture, 160 in order to check for parallel signatures of introgression that would be expected under tight 161 linkage to the simple sequence tandem repeat units. We reasoned that apparent introgression at 162 STRs but not at the adjacent flankers would be best explained by SH. 
165 loci (A9 and A122) in selected individuals of $M$. gracilis and M. recondita, including those with 166 possible mixed species ancestries. In total, ten bats from each of the two species were selected 167 (indicated by arrows in Fig. 1) plus one M. eleryi (HNHM 2007.28.2; see Table S2) for 168 comparative purposes. We designed and paired the primers A9FRL (5'-GCA ATT TCA TTG 169 TGT CCC TTG-3') and A9FRR (5'-GTC ATA GTT CTA GTC TCC CAG ATC C-3') for A9, 170 and A122FRL (5'-CAT TCT ATC TGC CTA CCT TGA CA-3') and A122FRR (5'-GGC CTT 171 CTC ACT AGG CAC AG-3') for A122. PCR cocktails of $15 \mu \mathrm{L}$, contained $0.2 \mu \mathrm{M}$ each primer, $172 \quad 2.0 \mu \mathrm{L}$ of template, and $7.5 \mu \mathrm{L}$ of the provided master mix of the Qiagen Type-it Microsatellite 173 PCR Kit (QIAGEN). Reactions were performed on a BIO-RAD C1000 Thermal Cycler (BIO174 RAD Laboratories) with the following thermal profile: $95^{\circ} \mathrm{C} 5 \mathrm{~min} ; 35$ cycles of $95{ }^{\circ} \mathrm{C} 30 \mathrm{sec}, 59$ $175{ }^{\circ} \mathrm{C} 90 \mathrm{sec}, 72{ }^{\circ} \mathrm{C} 30 \mathrm{sec} ; 60^{\circ} \mathrm{C} 30 \mathrm{~min}$. Sanger sequencing using primers A9FRR and A122FRR 176 was undertaken by Eurofins MWG Operon (Ebersberg, Germany) on an ABI 3730 DNA 177 Analyzer (Applied Biosystems).

Sequences were trimmed and the chromatograms inspected by eye for double peaks, 180 indicative of heterozygous sites. For reads with multiple heterozygous sites, we used the 181 Bayesian statistical method in PHASE 2.1.1 (Stephens et al. 2001; Stephens \& Scheet 2005) to 182 infer haplotypes. PHASE analysis was applied separately to $M$. gracilis and $M$. recondita, using 183 models that both allow or disallow intra-genic recombination. To facilitate phasing, known 184 haplotypes obtained from 454 sequencing were used as references (see next section). Under each 185 recombination model, we performed three replicated runs, each with 10,000 iterations (10 
186 iterations per sample) following a burn-in of 1000 iterations. Haplotypes inferred by PHASE with 187 a posterior probability of $\geq 0.6$ were accepted (see Garrick et al. 2010). To investigate the 188 segregation of haplotypes in relation to species identity, for each locus we constructed median189 joining (MJ) network using the software NETWORK 4.6.1.1 (Fluxus Technology Ltd. 2010) in 190 which the epsilon value was heuristically set as 10.

Divergence within the M. gracilis complex revealed by $m t D N A$

193 To obtain a more comprehensive picture of the divergence processes among M. gracilis, M. 194 recondita and their relatives, we further included 12 additional individuals of $M$. eleryi collected 195 from Southern China $(\mathrm{n}=7)$ and Central Vietnam $(\mathrm{n}=5)$ (see Table S2 for details). Genomic DNA 196 of these bats was extracted using the DNeasy Blood \& Tissue Kit (Qiagen).

We first investigated the divergence processes based on mtDNA by expanding our earlier 199 published dataset (Kuo et al. 2014; see Table 1) by Sanger sequencing the partial cytochrome $b$ 200 (Cyt- $b$ ) and cytochrome c oxidase subunit 1 (COI) genes of these additional M. eleryi samples, 201 which we also supplemented with published sequences (Francis et al. 2010; see Table 1). To 202 infer the order of divergence among M. gracilis, M. recondita and M. eleryi taxa we used Heled 203 and Drummond's (2010) Bayesian method implemented in *BEAST. Assuming the absence of 204 post-split gene flow between divergent taxa, *BEAST can assess the stochastic process of 205 coalescence by explicitly modeling effective population size along the course of divergence, and 206 has also been shown to perform very well in estimating the order and timing of divergence events, 207 even when applied to a single locus (see Drummond et al. 2012). The two mitochondrial genes 
208 were concatenated and partitioned, into the first and second codon positions together (partition

$\left.209 \mathrm{CP}_{12}\right)$ and the third codon position $\left(\mathrm{CP}_{3}\right)$. For $\mathrm{CP}_{12}$ and $\mathrm{CP}_{3}$, we used the nucleotide substitution

210 models HKY+I (HKY, Hasegawa et al. 1985) and GTR+I+G (GTR, Tavaré 1986), respectively,

211 selected based on the Akaike Information Criterion in jModelTest 2.1.3 (Posada 2008). The

212 demography along the course of divergence was modeled by constant-sized populations between

213 sequential splits, namely the piecewise-constant (PC) model. We used the extinction-free

214 constant-rate birth process (Yule 1924) as a prior for divergence, and, under a strict clock model,

215 we calibrated the root of the taxon tree with a lognormal prior of $\log (\operatorname{mean})=0.96$ and

$216 \log ($ standard deviation $)=0.15$, corresponding to an mtDNA-based estimate of the split time

217 between $M$. eleryi and the common ancestor of $M$. gracilis and M. recondita as 2.62 (95\% CI:

218 1.86-3.48) million years ago (Ma) (see Systematic notes on the focal species, Supporting

219 information). We performed two replicate MCMC runs in BEAST 1.7.4 (Drummond et al. 2012),

220 each with 100 million iterations (8,000 iterations per sample) and the first $10 \%$ of runs discarded

221 as burn-in. We examined the runs in Tracer 1.5 (Rambaut \& Drummond 2007) as well as with

222 the 'starbeast_demog_log' python script in the biopy 0.1 .7 package (available from:

$223 \mathrm{http} / /$ code.google.com/p/biopy/); these two runs gave consistent results and so were combined to

224 give an effective sample size (ESS) of $>2,600$ per parameter.

226 Nuclear markers for investigating divergence within the M. gracilis complex

227 For insights into the divergence process in the nuclear genome among the focal taxa, we 228 developed a set of ten anonymous autosomal markers based on microsatellite flanking regions 229 (A4, A9, A104, A109, A118, A122, B5, B114, B124 and D117). For each locus we designed new 
230 primer pairs based on clone sequences to amplify the flanker but to exclude the microsatellite

231 motif (Table S3, Supporting information). Primers pairs designed for A9 and A122 amplified

232 fragments that overlap with those described in the earlier section.

233

For M. gracilis and M. recondita, we obtained sequences for each of the ten loci by highthroughput 454-Pyrosequencing (Margulies et al. 2005). Given that both species show strong geographic substructure within Taiwan (Kuo et al. 2014), which would violate assumptions of coalescent-based analyses, sequences were only generated for 15 individuals of each taxon from part of their respective ranges (Table 1). To sort sequences, we incorporated unique 3-bp tags at the 5 ' end of the synthesized forward primer for each locus. For M. gracilis, we used the tags ACA, ACG, ACT, AGA, AGC, AGT, ATA, ATC, ATG, CGA, CGC, CGT, CTA, CTC and

CTG, while for M. recondita we used CAT, GTA, GTC, GTG, GAC, GAG, GAT, GCA, GCG,

GCT, TAC, TAG, TAT, TCA and TCG. PCR products of each locus were visualized on a gel and pooled for 454-Pyrosequencing at Mission Biotech (Taipei, Taiwan) on a 454 GS Junior System (Roche/454 Life Sciences).

We used the software CLC Genomics Workbench 4.9 (CLC bio, Denmark) to filter and sort 454-reads as follows. First, we used original clone sequences as references on which 454

248 reads were mapped with a coverage parameter of 0.6 and a similarity parameter of 0.9 . Second,

249 we sorted mapped sequences into individual bats on the basis of the 3-bp tag plus 2-3 sites of the $250 \quad 5^{\prime}$ end of the forward primer. Sorted sequences were aligned using MUSCLE (Edgar 2004) in 251 MEGA 5 (Tamura et al. 2011). For each individual, 50 reads were trimmed and aligned (in cases 
252 where fewer reads were available, $<50$ were used), and, assuming that error rates per nucleotide

253 were lower than correct base calls (Galan et al. 2010), we inferred the haplotypes of every bat for 254 each of its two chromosomes. We adopted the following conservative criteria for acceptance of 255 haplotypes for downstream genetic analyses: (1) for each individual, only inferred haplotypes 256 with frequencies of $\geq 5$ were accepted such that the occurrence of two haplotypes $\geq 5$ signified a 257 heterozygote, and (2) each individual was considered homozygous for a locus when only one 258 haplotype was detected with a frequency of $\geq 10$. Bats that did not meet these criteria, because 259 only one haplotype was detected at a frequency of $\geq 5$ but $<10$, were considered to be potentially 260 heterozygous but with missing data for one of the two chromosomes.

We also sequenced the same ten loci in M. eleryi using Sanger sequencing (Table 1), and 263 confirmed in a subset of individuals of $M$. gracilis and $M$. recondita that both sequencing 264 methods gave consistent results. PCR products were sequenced in both directions at Eurofins 265 MWG Operon. In four of the ten loci, indels (1-9 bp) were observed, with individuals 266 heterozygous for such indels showing characteristic double peaks in downstream stretches on 267 their chromatograms. In these individuals, we were able to deduce the nucleotide sequences of 268 each haplotype based on the rationale that the superposed signatures would correspond to two 269 nucleotides that differed by a length equal to that of the indel (Flot et al. 2006). Where two 270 haplotypes could not be inferred in this way we used PHASE (see earlier section), treating the 271 two clades of $M$. eleryi that were identified and are hereafter referred to as M. eleryi 1 and 2 (see 272 Results). 
Final alignments of each of the ten nuclear markers contained samples of M. gracilis, $M$.

275 recondita, M. eleryi 1 and $M$. eleryi 2. Excluding indels, we assessed the genetic variability of

276 each taxon at each locus based on the number of segregating sites, Watterson's (1975) theta value

277 and nucleotide diversity, all calculated in DnaSP 5.10.01 (Librado \& Rozas 2009). We also used

278 NETWORK to construct (epsilon = 10) locus-wise MJ genealogies for samples of all four taxa in

279 order to gain information on genetic differences among them. Polzin and Daneshmand's (2003)

280 maximum parsimony algorithm was used to remove unnecessary links in the network for one

281 locus (A122) for which complex connections were seen.

282

Prior to subsequent coalescent-based analyses, for each locus we tested for recombination using Hudson and Kaplan's (1985) four-gamete test assuming an infinite-site model of evolution, as implemented in the software IMgc (Woerner et al. 2007), in each case retaining the largest recombination-free block of sequences. This approach was not applied to locus A122, due to the detection of a tri-allelic segregating site that suggested this locus did not evolve in an infinite-site manner and might experience a faster mutation rate (also corroborated by analyses of genetic variability in the four focal taxa; see Results). Throughout this study, the HKY model was used 290 for nucleotide substitutions for A122.

292 Divergence among taxa within the M. gracilis complex revealed by ncDNA

293 The order of divergence among focal taxa was inferred in *BEAST, using the recombination-free 294 sequences from ten nuclear loci, and with a JC nucleotide substitution model (Jukes \& Cantor 295 1969) applied to each except for A122. For demographic analysis throughout divergence, we 

used the PC model (see Selection of a demographic model for the ncDNA *BEAST analysis,

297 Supporting information for details of model selection). For calibration of multi-locus coalescence 298 events, we specified for each locus a strict clock with the clock rate sampled from the following 299 lognormal prior: $\log ($ mean $)=-6.12, \log ($ standard deviation $)=0.58$. This prior was set for a mean 300 estimate of the neutral mutation rate of mammalian nuclear genomes as 0.0022 substitutions per 301 site per million years (Kumar \& Subramanian 2002) while allowing rates to vary among loci to 302 an order of magnitude. Two replicate *BEAST runs were combined to give an ESS of $>200$ per 303 parameter. Finally, a generation time for the focal taxa as two years was used to calculate the 304 effective population sizes from the estimated demographic parameters. gracilis), we then inferred the divergence process among these taxa in further detail, and 308 specifically to estimate gene flow among them, we used isolation-with-migration (IM) models 309 implemented in IMa2-8.26.11 (Hey \& Nielsen 2007; Hey 2010b). An infinite site model was 310 adopted for each of the ten loci analysed except for A122. Truncated uniform priors for entry 311 demographic parameters of IMa2 were set following the author's recommendations (Hey 2011); 312 specifically we set upper bounds of priors for the composite parameter of the split time (with the

313 flag $-\mathrm{t}$ ), the rescaled migration rate in either direction (-m), and the population mutation rate for 314 each of the extant/ancestral taxa (-q) based on the genetic variability of M. eleryi 1 , which had 315 highest genetic variability of the four species (see Results). The geometric mean of Watterson's 316 (1975) theta values over loci - an estimate for the population mutation rate - was 0.0055 per 317 nucleotide site, corresponding to a value of 1.1 per locus given a geometric mean of sequence 
318 lengths over the ten loci as 196 . We set $-\mathrm{t} 2$ and -q5 for two and five times of the above estimates,

319 respectively, and for post-split migrations we set $-\mathrm{m} 2$ for a moderately high upper bound for the

$3202 \mathrm{NM}$ value, since $2 \mathrm{NM}=\mathrm{q} \times \mathrm{m} / 2=1.1$ under the set ' $\mathrm{m}$ ' value. In addition, we explored a

321 different $\mathrm{m}$ prior, modeled by an exponential distribution with a mean value of 0.5 (with flags $-\mathrm{j} 8$

$322-\mathrm{m} 0.5)$, which circumvents truncation of the marginal estimate by an upper bound and is justified

323 under the expectation of low migration between diverging taxa (Hey 2010b).

We also applied IM models, with identical prior settings to those described above, to

326 pairwise datasets of the four focal taxa. Divergence times estimated independently for pairwise

327 taxa could inform the splitting order (Hey 2010a) and thus corroborate the taxon phylogeny

328 inferred by *BEAST. Metropolis-coupling MCMC $\left(\mathrm{MC}^{3}\right)$ for all above four- and two-taxon IM

329 runs was conducted with a geometric heating scheme for 60 chains (heating parameters -ha0 0.95 -

330 hb0.8), with one million iterations discarded as burn-in followed by five million iterations for

331 sampling (100 iterations per sample). Three (for four-taxon analyses) or two (for two-taxon

332 analyses) replicate runs were performed under each prior setting for assessment of convergence.

333 Given that replicate runs appeared to converge, we combined results under each specific setting

334 using the 'L mode' function of IMa2, and ran likelihood ratio tests (LRT) to assess evidence of

335 post-split gene flow between focal taxa, following Nielsen and Wakeley (2001). Finally, to

336 convert entry demographic parameters into interpretable scales, we applied a mutation rate of

3370.0022 per nucleotide site per million years (Kumar \& Subramanian 2002), a geometric mean of

338 sequence lengths of $196 \mathrm{bp}$, and a generation time of two years. 


\section{Results}

341 Assessment of contemporary gene flow between M. gracilis and M. recondita

342 Clustering analyses of 14 microsatellite loci yielded two different configurations at $\mathrm{K}=3$, and

343 also at $\mathrm{K}=4$ (Fig. 1). In each of these cases, lower 'penalized log-likelihood values' (Pritchard et

344 al. 2000) were obtained for a configuration with an empty cluster that contained no substantial

345 fraction of any individual bat of either species. By excluding runs with empty clusters (Guillot

346 2008) the penalized log-likelihood was highest at $\mathrm{K}=4$ in which four $M$. recondita individuals

347 (labeled as RA, RB, RC, and RD in Fig. 1) showed $>10 \%$ of their genetic composition appearing

348 to originate from M. gracilis. All other bats of both species were assigned almost exclusively to

349 genetic clusters of their own species (each with a sum of ancestry coefficients q for its own $350 \quad$ species $>0.9)$.

351 Repeated STRUCTURE analyses with each locus sequentially removed revealed that 352 apparent genetic admixture among some $M$. gracilis and $M$. recondita individuals was mainly 353 attributable to loci A9 and A122 (Fig. 1; Table S4, Supporting information). To determine if

354 these signatures stemmed from introgression, we sequenced the flanking regions of A9 and A122 355 in selected bats (shown by arrows in Fig. 1) and constructed median-joining (MJ) networks. In 356 the network based on A9, haplotypes of $M$. gracilis and $M$. recondita clustered by species, 357 however, $M$. recondita showed an unexpected affiliation with M. eleryi (Fig. 2). Similarly, for the 358 A122 network, all $M$. recondita haplotypes clustered together with the exception of one that 359 grouped with $M$. eleryi (Fig. 2). It follows that apparent signatures of genetic admixture between

360 M. gracilis and $M$. recondita seen in multi-locus microsatellite genotypes were not supported by 361 analyses of these microsatellites' flanker sequences, suggesting they reflect microsatellite allele 
362 size homoplasy rather than true introgression. At the same time, however, we discovered an

363 unexpectedly close relationship between $M$. recondita and $M$. eleryi that could be due to

364 introgression. Based on this finding, we expanded sampling of M. eleryi in subsequent analyses

365 to dissect the divergence history among all these Murina species.

366

367 Divergence within the M. gracilis complex revealed by $m t D N A$

368 Using *BEAST, the concatenated mitochondrial sequences of Cyt- $b$ and COI (Table 1) recovered

369 a mtDNA genealogy (not shown) with four maximally supported monophyletic groups (posterior

370 probability $=1$ ) representing $M$. gracilis, $M$. recondita, $M$. eleryi from Southern China (M. eleryi

371 1) and M. eleryi from Central Vietnam (M. eleryi 2). Among these lineages, M. gracilis and $M$.

372 recondita grouped together with maximal support, as did the two $M$. eleryi lineages. These

373 relationships were also recovered in the inferred species tree (posterior probabilities $>0.99$; see

374 Fig. 3a). These two split events had median estimates of 0.79 (95\% CI: $0.31-1.29)$ and 0.43 (95\%

375 CI: 0.10-0.77) Ma, respectively (Fig. 3a). Effective population sizes for extant and ancestral taxa

376 ranged from 51,000 (M. eleryi 1) to 250,000 (M. recondita) as shown in Figure 3 a.

$378 n c D N A$ structure within the M. gracilis complex

379 454-Pyrosequencing of autosomal microsatellite flanker sequences in larger sample sets of $M$.

380 gracilis and $M$. recondita generated 73,395 reads, which were assembled to yield haplotypes for

381 all ten target loci from both chromosomes in all but one individual of $M$. gracilis and $75 \%$ of

382 individuals of $M$. recondita. Sanger sequencing of orthologous sequences in $M$. eleryi yielded

383 haplotypes from both chromosomes in $70 \%$ of individuals (see Table 1). 
Locus-specific MJ networks supported pilot sequencing of two loci, confirming that $M$. recondita ncDNA sequences were typically more related to those of $M$. eleryi than to those of $M$.

387 gracilis (Fig. S1, Supporting information). For three of ten loci, M. recondita shared haplotypes 388 with the two M. eleryi lineages but not with M. gracilis, and in cases where M. recondita shared 389 haplotypes with $M$. gracilis, these haplotypes were also shared by the two M. eleryi lineages. At 390 most loci, M. eleryi 1 and $M$. eleryi 2 had contrasting haplotype composition, indicative of 391 divergence. Locus-wise measures of genetic variability for the four taxa are shown in Table 2. M. 392 recondita had a lower level of genetic variability than all the others, particularly reflected by its 393 lower $\theta_{\mathrm{S}}$ and $\pi$ values at most of the ten markers. Four-gamete tests suggested potential intra394 locus recombination at loci A4, A104, A109 and B124, for which relevant sections of alignments 395 (for A4 and A109) or individual sequences (for A104 and B124) were removed using IMgc 396 (shown in Fig. S1).

Divergence in M. gracilis complex revealed by $n c D N A$

399 Supporting the haplotype networks, the species tree reconstructed using *BEAST recovered a 400 monophyletic clade containing $M$. recondita and both $M$. eleryi taxa (posterior probability $=1$ ), 401 which was estimated to have diverged from M. gracilis around 2.06 (95\%CI: 1.13-3.19) Ma (Fig. 402 3b). Within the (M. recondita $+M$. eleryi) clade, $M$. recondita formed a moderately well 403 supported clade with M. eleryi 1 (posterior probability $=0.79$ ) to the exclusion of M. eleryi 2. 404 The split between $M$. eleryi 2 and the common ancestor of $M$. recondita and $M$. eleryi 1 was 405 dated to $0.66(95 \% \mathrm{CI}: 0.28-1.14) \mathrm{Ma}$, while divergence between the latter two taxa was dated to 
$406 \quad 0.42$ (95\%CI: 0.16-0.77) Ma. Effective population sizes of lineages under the PC model ranged 407 from 59,000 (M. recondita) to 632,000 (common ancestor of M. recondita and the two M. eleryi 408 taxa) (see Fig. 3b).

To aid descriptions, hereafter we use the abbreviations 4PM2 and 4PME0.5 for four-taxon

411 isolation-with-migration (IM) analyses specifying a truncated uniform prior (upper bound $=2$ )

412 and an exponential prior $($ mean $=0.5)$, respectively. For focal demographic parameters estimated

413 with the 4PM2 prior, we show summary statistics in Figure 4 and present marginal distributions

414 in Figure S2 (Supporting information). Summary statistics of these demographic parameters from 415 both 4PM2 and 4PME0.5 analyses are also presented in Table S5 (Supporting information). The 416 marginal distribution for the post-split migration parameter, $\mathrm{m}$, was more compressed toward 417 zero with 4PME0.5 than with 4PM2 prior, leading to a smaller estimate from 4PME0.5 analysis 418 (a trend also reflected in the population migration rate, 2NM; see Table S5); this was true for all $419 \mathrm{~m}$ parameters along the course of divergence although such compressions in marginal 420 distributions were less apparent for those with peaks at zero. For all other demographic 421 parameters including times of split and effective population sizes, similar estimates were obtained 422 regardless of the prior used (Table S5).

From both 4PM2 and 4PME0.5 analyses, modal estimates for times of split indicated 425 divergence between $M$. recondita and M. eleryi 1 (T1), between the common ancestor of these 426 two taxa and M. eleryi 2 (T2), and between the common ancestor of three taxa and M. gracilis 427 (T3), at 1.2-1.4, 1.8-1.9, and around 2.3 Ma, respectively. Nevertheless, confidence sets for these 
428 parameters were rather broad (see Fig. 4 for values from 4PM2 analysis); this was especially true

429 for T1 and T3 for which flat marginal distributions were obtained (see Fig. S2a for curves from $4304 \mathrm{PM} 2$ analysis). Among a total of $18 \mathrm{~m}$ parameters, seven had non-zero modes under 4PM2: bi431 directional migration between $M$. recondita and $M$. eleryi 1 , and migration from $M$. recondita to 432 M. eleryi 2, from $M$. eleryi 2 to $M$. eleryi 1 , from $M$. eleryi 2 to the common ancestor of $M$. 433 recondita and $M$. eleryi 1 , from the common ancestor of $M$. recondita and $M$. eleryi 1 to $M$. 434 gracilis, and from the common ancestor of $M$. recondita and both $M$. eleryi taxa to M. gracilis 435 (Fig. S2b). Of these seven, only the former four also had non-zero modes under 4PME0.5. Based 436 on either $\mathrm{m}$ or 2NM, LRTs were significant for migrations from $M$. recondita to M. eleryi 1 and 437 from $M$. eleryi 2 to $M$. eleryi 1 under both priors except for migration from $M$. recondita to $M$. 438 eleryi 1 based on $\mathrm{m}$ under 4PME0.5. Non-significant results were obtained for all other LRTs 439 (see Table S5 for a summary of LRTs based on 2NM). Under the two priors used, effective 440 population sizes for the extant taxa had modal estimates ranged from 27,000/33,000 (M. 441 recondita) to 322,000/348,000 (M. eleryi 1) or 319,000/349,000 (M. eleryi 2) (Fig. 4; Table S5). 442 For effective population sizes of ancestral populations, flat marginal distributions (Fig. S2c), and 443 thus broad confidence intervals (Table S5), were obtained, suggesting little information from the 444 data on these parameters.

As in the four-taxon IM analyses, we used the abbreviations 2PM2 and 2PME0.5 for twotaxon IM analyses specifying a truncated uniform prior (upper bound $=2$ ) and an exponential 448 prior $($ mean $=0.5)$, respectively. Summary statistics for all six pairs of taxa analysed under 2 PM2 449 prior are shown in Figure 5 while their marginal distributions are presented in Figure S3 
450 (Supporting information). A full list of summary statistics for all two-taxon analyses under the

451 two priors used is given in Table S6 (Supporting information). Again, the marginal distribution

452 for each m parameter was more compressed toward zero under 2PME0.5 than under 2PM2 prior,

453 leaving smaller $\mathrm{m}$ estimates in the former analysis (see Table S6, Supporting information). For

454 parameters of split times and effective population sizes, on the other hand, IM estimates were

455 insensitive to the prior used (Table S6).

From two-taxon IM analyses, comparing modal estimates across datasets suggested that

458 the order of splits among taxa was consistent with the results from ncDNA-based *BEAST; thus

$459 M$. recondita and M. eleryi 1 split most recently (1.1-1.2 Ma), then M. eleryi 2 and either $M$.

460 recondita or $M$. eleryi 1 (1.4-1.5 Ma), and finally the most ancient split was between $M$. gracilis

461 and either of the two M. eleryi lineages (1.5-1.7 Ma) (see Fig. 5 for values from 2PM2 analyses).

462 Inconsistent with this inferred splitting order, modal estimates from two-taxon IM analyses

463 suggested an unexpected recent divergence between $M$. gracilis and M. recondita (1.2-1.3 Ma).

464 However, under either of the two priors used, the marginal distribution for the split time of $M$.

465 gracilis and $M$. recondita was the flattest one among the six pairwise analyses (see Fig. S3a for

466 curves from 2PM2 analyses), implying greater uncertainty underlying this estimate. When $M$.

467 gracilis was paired with either of the other taxa, modal estimates of $\mathrm{m}$ were zero for both

468 directions. In contrast, modal estimates of $\mathrm{m}$ for the remaining pairwise combinations of taxa

469 were non-zero for both directions (Fig. S3b). Based on either m or 2NM, LRTs were significant

470 for bi-directional migrations between $M$. recondita and $M$. eleryi 1 and migration from M. eleryi

4712 to $M$. recondita under both priors except for migration from $M$. recondita to $M$. eleryi 1 based 
472 on m under 2PME0.5. LRTs were also significant for bi-directional migrations between M. eleryi

4731 and $M$. eleryi 2 based on 2NM under 2PME0.5 (Table S6). Two-taxon IM analyses gave 474 effective population size estimates for $M$. gracilis, M. recondita, and M. eleryi 2 broadly 475 consistent with those from four-taxon analyses. In contrast, the estimates for M. eleryi 1 were 476 higher than obtained from four-taxon analyses (Table S6). As with the four-taxon models, two477 taxon analyses gave diffuse marginal distributions for effective population sizes of ancestral 478 populations (Fig. S3c), suggesting limited information about these parameters was available from 479 the data. 
482 Cases of geographical confinement of sister taxa to islands are rare, and have been proposed as 483 candidate systems in which non-allopatric speciation processes could have occurred (White 1978;

484 Coyne \& Price 2000; Coyne 2007). Here we examined the process of divergence between the 485 tube-nosed bats Murina gracilis and M. recondita, both of which are endemic to Taiwan and 486 which, based on phylogenetic reconstructions of mtDNA, appear to be sister species (Kuo 2004, 2013). To determine whether these taxa did indeed form in the face of migration, consistent with non-allopatric speciation, we tested for gene flow at different temporal scales using a variety of

489 molecular markers and statistical approaches. Tests for contemporary gene flow based on ncDNA 490 uncovered an unexpected sister relationship between $M$. recondita and a continental species $M$. 491 eleryi, to the exclusion of $M$. gracilis. The demographic process and putative mechanisms 492 responsible for this mtDNA-ncDNA conflict are discussed below.

494 Absence of recent introgressive hybridization between M. gracilis and M. recondita

495 Although, for the most part, microsatellite-based clustering suggested little, if any, contemporary 496 gene flow between $M$. gracilis and M. recondita (250 individual bats in total; Fig. 1), a small 497 number of individuals of $M$. recondita did appear to show mixed ancestry with M. gracilis (Fig. 1

498 and Table S4). Such cases of apparent genetic admixture based on microsatellite clustering are 499 not unusual, and are typically attributed to introgression or the retention of ancestral 500 polymorphism (e.g. Muir \& Schlötterer 2005; Berthier et al. 2006; Randi 2008; Brown et al. 501 2010; Bogdanowicz et al. 2012). In our study, however, sequencing and analyses of the 502 respective $M$. recondita microsatellite flanking regions - which are expected to evolve more 
503 slowly than their adjacent microsatellite motifs (Estoup et al. 2002) - did not support admixture.

504 Instead, in networks based on these flanking regions, the two putative sister taxa were 505 reciprocally monophyletic with respect to each other (Fig. 2). It follows that apparent admixture 506 signatures based on microsatellite genotyping appear to stem from allele size homoplasies, a 507 well-described phenomenon that is nonetheless infrequently tested for and described in studies of 508 population structure (exceptions include Adams et al. 2004; Rossiter et al. 2007).

\section{Divergence between $M$. gracilis and M. recondita}

511 Further clustering analyses of ten microsatellite flanking sequences, in which both focal taxa

512 were included along with the mainland congener $M$. eleryi, recovered an unexpected grouping of

513 the latter with $M$. recondita. Specifically, phylogenetic reconstruction performed in *BEAST - an

514 approach that simultaneously reconstructs the gene tree(s) and evaluates the support of different

515 species trees in generating such a gene tree (or gene trees) through stochastic drift (Heled \&

516 Drummond 2010; Drummond et al. 2012) - indicated that M. recondita formed a monophyletic

517 clade with $M$. eleryi 1 from Southern mainland China, which in turn formed a clade with $M$.

518 eleryi 2 from Central Vietnam to the exclusion of M. gracilis (Fig. 3b). Additional supporting

519 evidence for this history of divergence came from ncDNA-based two-taxon isolation-with-

520 migration (IM) analyses (Fig. 5; Fig. S3a) by which split times of divergent taxa were estimated

521 while accounting for the potential influences of post-split gene flow (Hey \& Nielsen 2004; Hey

$522 \&$ Nielsen 2007). It is important to recognize that these estimated split times had broad

523 confidence intervals, indicative that the data contains limited information about these parameters.

524 Further uncertainty in these estimates arises from the fact that they were solely informed by a 
525 mean multi-locus mutation rate. Despite uncertainty about these split times, however, it is

526 noteworthy that the IM and *BEAST analyses of microsatellite flanking sequences both

527 suggested the same order of divergence; thus the mitochondrial and nuclear genomes harbored

528 conflicting signals about the divergence process of the focal taxa.

By superimposing the mtDNA genealogy for the four focal taxa on the splitting order

531 obtained by the ncDNA, we were able to infer a scenario of independent incursions of the

532 Taiwanese species. M. gracilis appears to have colonized first, followed by $M$. recondita with

533 massive mtDNA introgression from the former to the latter. The clustering of range-wide mtDNA

534 haplotypes of both Taiwanese species to the exclusion of those from $M$. eleryi implies that the

535 mitochondrial genomes of $M$. recondita have been completely replaced by those of M. gracilis.

536 Meanwhile, the present reciprocally monophyletic relationship between the two Taiwanese

537 species indicates that any introgression has since ceased, probably around 0.3-1.3 (median: 0.8)

538 Ma (Fig. 3a: split between M. gracilis and M. recondita). In contrast, neither the two-taxon nor

539 four-taxon IM analyses detected post-split nuclear gene flow between M. gracilis and $M$.

540 recondita (Fig. 4 and Fig. 5, respectively).

542 Like all species of their genus, M. gracilis and M. recondita show a suite of eco543 morphological adaptations for living in the forest interior, including broad wings and very large 544 call bandwidths for detecting arthropods in dense clutter (Kingston et al. 1999; Kingston et al. 545 2003; Schmieder et al. 2010). At the same time, however, such traits are associated with reduced 546 gene flow (Struebig et al. 2011) and are likely to constrain the ability of these bats to move 
547 across more open environments, including water bodies such as the Taiwan Strait. It follows that

548 the two inferred incursions will almost certainly have occurred during glacial periods when the 549 drop in sea-level exposed the continental shelf connecting Taiwan and mainland Asia (Voris 550 2000). Consequently rather than diverging non-allopatrically on Taiwan, as we initially 551 hypothesized based on their restricted island distributions, M. gracilis and M. recondita appear to 552 have speciated during a period of geographical isolation, with their co-distribution arising via 553 secondary contact. Similar histories of double incursions for pairs of congeneric Taiwanese taxa 554 have been inferred in rodents (genera Apodemus and Niviventer; Yu 1995) and grass lizards 555 (genus Takydromus; Lue \& Lin 2008), both of which showed a paraphyletic relationships among 556 island taxa with respect to continental forms.

Several scenarios may account for the observed massive introgression of mtDNA, yet 559 little or no introgression of ncDNA, from $M$. gracilis to $M$. recondita. First, mitochondrial 560 replacement can arise due to positive selection (e.g. reviewed in Melo-Ferreira et al. 2014). 561 Despite finding no evidence of positive selection at mtDNA, we cannot rule this out (Tests of 562 positive selection, Supporting information), especially given that mitochondrial protein-coding 563 genes are subject to genetic hitch-hiking due to a lack of recombination that characterizes 564 mitogenomes (Galtier et al. 2009). Alternatively, contrasting levels of mtDNA and ncDNA 565 introgression can also reflect asymmetries between species in the extent to which they undergo 566 assortative mating, or experience sex-biased reductions in hybrid fitness (Chan \& Levin 2005;

567 Mallet 2005). Perhaps the simplest explanation for the observed discordance between mtDNA 568 and ncDNA markers lies in the fact that the two Taiwanese species would have experienced 
569 different demographic conditions when they colonized Taiwan, as expected given the inferred 570 scenario of two temporally distinct incursions. Specifically, it is probable that during the

571 incursion of Taiwan by $M$. recondita, this colonizing taxon will have been in a phase of relative 572 demographic growth compared to the earlier arriving M. gracilis. Simulations by Currat et al. 573 (2008) show that under such conditions, introgression occurs from resident to the colonizing 574 population. While massive mtDNA introgression from $M$. gracilis to $M$. recondita could have 575 occurred via such 'allele surfing', a lack of parallel ncDNA introgression could be attributed to 576 male-biased gene flow among conspecific populations of the latter species (Tests for sex-biased 577 dispersal in Murina gracilis and M. recondita, Supporting information), so that M. gracilis 578 nuclear alleles would have faced competition from those of conspecific populations, and thus 579 reduced their chance of fixation. Indeed, evidence for the influence of sex-biased dispersal on 580 differential rates of introgression among markers with different modes of inheritance has been 581 reported in a wide range of taxa (Petit \& Excoffier 2009). Further studies are needed to 582 disentangle the contributions of these potential mechanisms between $M$. gracilis and $M$. 583 recondita.

585 Introgression in tube-nosed bats and other bat species

586 Introgression was not only inferred for M. gracilis to M. recondita. Our four-taxon IM analyses 587 also suggested post-split nuclear gene flow from $M$. recondita to the continental M. eleryi, as well 588 as between divergent clades of $M$. eleryi (Fig. 4). We therefore speculate that levels of mtDNA 589 introgression might be even higher in these cases, based again on insights and expectations from 590 simulations (Currat et al. 2008). In the former of these cases, more sampling of $M$. eleryi 
591 populations close to Taiwan is needed to test for mtDNA. In the latter case, on the other hand,

592 complete mitochondrial replacement between the two $M$. eleryi clades is implied by the

593 observation that the estimated split times based on both two- and four-taxon models were

594 substantially earlier than those estimated from mitochondrial genes. Unlike the situation between

595 M. gracilis and $M$. recondita where mitochondrial replacement occurred over only tens of

596 kilometres, any replacement between groups of $M$. eleryi would have involved much larger

597 geographic distances of up to $900 \mathrm{~km}$. Given that levels of introgression into invading species are

598 known to fall with distance from the front wave of hybridization (Currat et al. 2008), it is likely

599 that mitochondrial introgression in our focal bats is very efficient, and probably facilitated by

600 very low mtDNA gene flow among local populations (demonstrated in Kuo et al. 2014 for the

601 two Taiwanese species).

602

603

Our study of Murina sister taxa adds to the mounting evidence for introgression in bats.

604 Moreover, the inferred patterns - namely unidirectional rampant mtDNA introgression with little

605 or no nuclear introgression - show similarities to those reported for species of the genera Myotis

606 (Berthier et al. 2006) and Rhinolophus (Mao et al. 2013) as well as members of the family

607 Pteropodidae (Nesi et al. 2011). Higher introgression of mtDNA than of ncDNA has also been

608 implicitly suggested in other bat species (Larsen et al. 2010; Vallo et al. 2011), whereas higher

609 levels of introgression at ncDNA than at mtDNA in bats appears to be much rarer (but see Hulva

610 et al. 2010; Mao et al. 2010). Like in the case of Murina, Berthier et al. (2006) attributed

611 elevated mtDNA introgression in Myotis to contrasting demographic dynamics between the

612 invading versus resident species, together with male-based gene flow among populations of the 
613 former taxon. However, it is important to recognize that multiple alternative scenarios might

614 account for such patterns, leading Toews and Brelsford (2012) to call for a shift away from

615 documenting further mito-nuclear discordance towards hypothesis testing to rule out some

616 explanations of common patterns.

617

618 Conclusions

619 Our analyses of gene flow at different temporal and spatial scales indicate that rather than

620 diverging on Taiwan, the two endemic and closely related Murina species are a product of

621 vicariant speciation from a period of isolation. Consequently the current range overlap stems

622 from multiple incursions, while the previously accepted sister-relationship based on mtDNA is

623 incorrect and was obscured by mitochondrial introgression coupled with a lack of wider sampling

624 of geographically-distant continental taxa. This example reveals how historical processes of

625 vicariance, colonization and hybridization can readily lead to misleading signatures of non-

626 allopatric speciation, thus highlighting the need for careful analyses to distinguish among such

627 scenarios. Further caution comes from simulations of bird range data that show that even true

628 sister taxa that have speciated in allopatry can readily show partial range overlap and,

629 occasionally, complete range overlap (Phillimore et al. 2008). It is perhaps unsurprising,

630 therefore, that many of the most plausible cases of divergence with gene flow come from plants,

631 attributed to their greater tendency for fine-scale niche divergence (Anacker \& Strauss 2014).

632 Nonetheless, even in the most convincing and well-studied such examples it is still not trivial to

633 rule out the alternative explanation of double colonisations, particularly where introgression,

634 extinction and/or incomplete sampling are also possibilities (see Papadopulos et al. 2011). 
Table 1 Sample sizes, sources and sequencing methods (Sanger or 454Pyrosequencing) of genetic markers used for reconstruction of divergence processes among the focal taxa.

\begin{tabular}{|c|c|c|c|c|c|}
\hline \multirow[b]{2}{*}{ Taxon } & \multicolumn{3}{|c|}{ Mitochondrial markers } & \multicolumn{2}{|c|}{ Nuclear markers } \\
\hline & $\mathrm{n}$ & Cyt- $b$ & $\mathrm{COI}$ & $\mathrm{n}$ & FR \\
\hline M. gracilis & $87^{\mathrm{c}}$ & $\begin{array}{c}\mathrm{II}^{\mathrm{c}} \\
\text { Sanger }\end{array}$ & $\begin{array}{c}\mathrm{II}^{\mathrm{c}} \\
\text { Sanger }\end{array}$ & $15^{\mathrm{e}}$ & $\begin{array}{c}\mathrm{I} \\
454\end{array}$ \\
\hline M. recondita & $113^{\mathrm{c}}$ & $\begin{array}{c}\mathrm{II}^{\mathrm{c}} \\
\text { Sanger }\end{array}$ & $\begin{array}{c}\mathrm{II}^{\mathrm{c}} \\
\text { Sanger }\end{array}$ & $15^{\mathrm{e}}$ & $\begin{array}{c}\mathrm{I} \\
454\end{array}$ \\
\hline M. eleryi $1^{\mathrm{a}}$ & 8 & $\begin{array}{c}\text { I } \\
\text { Sanger }\end{array}$ & $\begin{array}{l}\mathrm{I} \text { III }^{\mathrm{d}} \\
\text { Sanger }\end{array}$ & 7 & $\begin{array}{c}\text { I } \\
\text { Sanger }\end{array}$ \\
\hline M. eleryi $2^{\mathrm{b}}$ & 5 & $\begin{array}{c}\text { I } \\
\text { Sanger }\end{array}$ & $\begin{array}{l}\mathrm{I} \text { III }^{\mathrm{d}} \\
\text { Sanger }\end{array}$ & 5 & $\begin{array}{c}\text { I } \\
\text { Sanger }\end{array}$ \\
\hline
\end{tabular}

$\mathrm{N}$, sample size; FR, flanking region sequence obtained for ten microsatellite markers; I, this study; II, Kuo et al. (2014); III, Francis et al. (2010).

a $M$. eleryi 1 refers to the single sample from North Vietnam (HNHM 2007.28.2) and samples from Southern China, listed in Table S2 (Supporting information).

${ }^{\mathrm{b}}$ M. eleryi 2 refers to samples from Central Vietnam, listed in Table S2.

${ }^{c}$ Range-wide samples for which concatenated Cyt- $b$ and COI sequences are available with Dryad entry doi:10.5061/dryad.f5th5.

d Available with accession numbers HM540936-7, JQ601475, JQ601483, JQ601503 and JQ601510 for M. eleryi 1, and HM540933, HM540938, JQ601543 and JQ601545 for M. eleryi 2.

e Selected samples from sites \#49 and \#54 for M. gracilis and those from sites \#48, \#51, \#52, and \#54 for M. recondita (see Kuo et al. 2014 for details of site locations). 
Table 2 Genetic variability of four taxa in the Murina gracilis complex based on ten microsatellite flanking regions. Values are calculated for each alignment showing no signature of intra-locus recombination except for the locus A122. Estimates of $\theta_{\mathrm{S}}$ and $\pi$ are presented as corresponding raw values multiplied by 100 .

\begin{tabular}{|c|c|c|c|c|c|c|c|c|c|c|c|c|c|c|c|c|c|}
\hline \multirow[b]{2}{*}{ Locus } & \multirow[b]{2}{*}{$\mathrm{L}$} & \multicolumn{4}{|c|}{ M. gracilis } & \multicolumn{4}{|c|}{ M. recondita } & \multicolumn{4}{|c|}{ M. eleryi 1} & \multicolumn{4}{|c|}{ M. eleryi 2} \\
\hline & & Nseq & $\mathrm{S}$ & $\theta_{\mathrm{S}}$ & $\pi$ & Nseq & $\mathrm{S}$ & $\theta_{\mathrm{S}}$ & $\pi$ & Nseq & $\mathrm{S}$ & $\theta_{\mathrm{S}}$ & $\pi$ & Nseq & $\mathrm{S}$ & $\theta_{\mathrm{S}}$ & $\pi$ \\
\hline A4 & 216 & 30 & 4 & 0.47 & 0.48 & 27 & 2 & 0.24 & 0.22 & 14 & 2 & 0.29 & 0.19 & 10 & 7 & 1.15 & 1.12 \\
\hline A9 & 102 & 29 & 3 & 0.75 & 1.22 & 15 & 0 & 0 & 0 & 14 & 3 & 0.93 & 0.97 & 10 & 2 & 0.69 & 0.55 \\
\hline A104 & 156 & 30 & 3 & 0.49 & 0.93 & 25 & 0 & 0 & 0 & 9 & 4 & 0.94 & 0.68 & 10 & 1 & 0.23 & 0.13 \\
\hline A109 & 137 & 30 & 2 & 0.37 & 0.45 & 30 & 1 & 0.18 & 0.05 & 14 & 3 & 0.69 & 0.40 & 6 & 2 & 0.64 & 0.49 \\
\hline A118 & 180 & 30 & 4 & 0.56 & 0.59 & 21 & 1 & 0.15 & 0.24 & 12 & 4 & 0.74 & 0.37 & 8 & 4 & 0.86 & 1.03 \\
\hline A122 & 184 & 30 & 5 & 0.69 & 0.63 & 24 & 7 & 1.02 & 0.86 & 6 & 10 & 2.38 & 2.86 & 10 & 7 & 1.35 & 1.88 \\
\hline B5 & 286 & 30 & 0 & 0 & 0 & 24 & 0 & 0 & 0 & 12 & 1 & 0.12 & 0.06 & 10 & 1 & 0.12 & 0.17 \\
\hline B114 & 318 & 30 & 0 & 0 & 0 & 23 & 1 & 0.09 & 0.16 & 12 & 2 & 0.21 & 0.27 & 8 & 4 & 0.49 & 0.60 \\
\hline B124 & 249 & 30 & 0 & 0 & 0 & 20 & 0 & 0 & 0 & 13 & 4 & 0.52 & 0.45 & 10 & 2 & 0.28 & 0.27 \\
\hline D117 & 234 & 30 & 4 & 0.43 & 0.42 & 29 & 0 & 0 & 0 & 12 & 5 & 0.71 & 0.86 & 8 & 5 & 0.82 & 0.93 \\
\hline
\end{tabular}

L, sequence length in number of base pairs; Nseq, sample sizes of sequences; S, number of segregating sites; $\theta_{\mathrm{S}}$, Watterson's (1975) theta; $\pi$, nucleotide diversity. 


\section{Figure captions}

637 Figure 1 Bayesian clustering analyses implemented in STRUCTURE. The upper panel shows 638 penalized log-likelihood values (Pritchard et al. 2000) of ten replicated runs under each of successive numbers of clusters $(\mathrm{K})$ for the full dataset (14 microsatellite loci). The lower panel shows DISTRUCT plots for analyses based on the full dataset and based on 13 loci with either locus A9 or locus A122 removed. Selected individuals for sequencing of flanking regions of loci A9 and A122 are indicated by arrows.

644

Figure 2 Median-joining networks based on flanking regions of microsatellite loci A9 and A122. Circles are coloured to represent haplotypes of Murina gracilis (yellow), M. eleryi (light blue), four individuals of $M$. recondita labeled as $\mathrm{RA}, \mathrm{RB}, \mathrm{RC}$, and $\mathrm{RD}$ in Figure 1 (grey), and remaining individuals of $M$. recondita (dark blue). Sizes of circles are proportional to sample sizes of unique haplotypes. The scale bar applies to both networks.

Figure 3 Divergence within the Murina gracilis complex inferred from *BEAST analyses based on (a) mtDNA and (b) ncDNA. In each panel, extant taxa are labeled as G for M. gracilis, $\mathrm{R}$ for $M$. recondita, E1 for $M$. eleryi 1 and E2 for $M$. eleryi 2 ; the upper left corner shows the maximum clade credibility (MCC) topology with posteriors for specific taxon grouping, and the lower right corner shows demographic estimates obtained under corresponding MCC topology. In the demographic plots, horizontal and vertical dimensions are scaled to represent effective population sizes (boxes) and split times (horizontal lines; present time at the top), respectively. Black boxes and black horizontal lines are scaled to represent median estimates, while grey ones including lines with double arrows are scaled to present 95\% confidence intervals of corresponding variables. Scale bars for 100,000 individuals and for one million years apply to both panels.

Figure 4 Divergence within the Murina gracilis complex inferred from four-taxon IM analyses under the 4PM2 prior. Extant taxa are labeled as in Figure 3 while ancestral populations are labeled as A1 for $M$. recondita plus $M$. eleryi 1 , A2 for $M$. recondita plus both $M$. eleryi taxa, and A3 for all extant taxa. Split times are numbered to present successive divergence events from recent to ancient ones under the specified phylogeny - (((R, E1), E2), G). Horizontal and vertical dimensions are scaled as in Figure 3 except that black boxes and black horizontal lines are scaled to present modal estimates. Black curved arrows mark directions of post-split migrations, each of which was significant using the population migration rate (2NM) based on Nielsen and Wakeley's (2001) likelihood ratio test; modal estimates of these 2NM values are also given.

667 Figure 5 Divergence within the Murina gracilis complex recovered by pairwise two-taxon IM analyses under the 2PM2 prior. For an explanation of the labels used and format of the plots, see Figure 4. Scale bars for 100,000 individuals and for one million years apply to all six plots. 
We thank Ian Barnes, Michael Bruford, Kalina Davies and Richard Nichols for helpful comments on this work. HCK was supported by the Overseas Research Students Awards Scheme and the University of London Central Research Fund as well as by the Studying Abroad Scholarship from the Taiwanese Ministry of Education. GC received support from the SYNTHESYS Project, which is financed by the European Community Research Infrastructure Action under the FP7 "Capacities" Program, and from the Hungarian Scientific Research Fund (OTKA) K112440. This research was also funded by a grant to SFC, YPF and SJR from the Taiwanese Ministry of Science and Technology (NSC 100-2621-B-126 -001).

\section{References}

Adams RI, Brown KM, Hamilton MB (2004) The impact of microsatellite electromorph size homoplasy on multilocus population structure estimates in a tropical tree (Corythophora alta) and an anadromous fish (Morone saxatilis). Molecular Ecology, 13, 2579-2588.

Anacker BL, Strauss SY (2014) The geography and ecology of plant speciation: range overlap and niche divergence in sister species. Proceedings of the Royal Society B-Biological Sciences, 281.

Barraclough TG, Vogler AP (2000) Detecting the geographical pattern of speciation from species-level phylogenies. American Naturalist, 155, 419-434.

Berthier P, Excoffier L, Ruedi M (2006) Recurrent replacement of mtDNA and cryptic hybridization between two sibling bat species Myotis myotis and Myotis blythii. Proceedings of the Royal Society B-Biological Sciences, 273, 3101-3109.

Bogdanowicz W, Piksa K, Tereba A (2012) Hybridization hotspots at bat swarming sites. Plos One, 7, e53334.

Brown RM, Nichols RA, Faulkes CG, et al. (2010) Range expansion and hybridization in Round Island petrels (Pterodroma spp.): evidence from microsatellite genotypes. Molecular Ecology, 19, 3157-3170.

Chan KMA, Levin SA (2005) Leaky prezygotic isolation and porous genomes: Rapid introgression of maternally inherited DNA. Evolution, 59, 720-729.

Coyne JA (2007) Sympatric speciation. Current Biology, 17, R787-R788.

Coyne JA, Price TD (2000) Little evidence for sympatric speciation in island birds. Evolution, 54, 2166-2171.

Currat M, Ruedi M, Petit RJ, Excoffier L (2008) The hidden side of invasions: massive introgression by local genes. Evolution, 62, 1908-1920.

Dieckmann U, Doebeli M (1999) On the origin of species by sympatric speciation. Nature, $\mathbf{4 0 0}$, 354-357.

Drummond AJ, Suchard MA, Xie D, Rambaut A (2012) Bayesian phylogenetics with BEAUti and the BEAST 1.7. Molecular Biology and Evolution, 29, 1969-1973.

Edgar RC (2004) MUSCLE: multiple sequence alignment with high accuracy and high throughput. Nucleic Acids Research, 32, 1792-1797.

Eger JL, Lim BK (2011) Three new species of Murina from southern China (Chiroptera: Vespertilionidae). Acta Chiropterologica, 13, 227-243. 
713

714

715

716

717

718

719

720

721

722

723

724

725

726

727

728

729

730

731

732

733

734

735

736

737

738

739

740

741

742

743

744

745

746

747

748

749

750

751

752

753

754

755

756

757
Estoup A, Jarne P, Cornuet JM (2002) Homoplasy and mutation model at microsatellite loci and their consequences for population genetics analysis. Molecular Ecology, 11, 1591-1604.

Fitzpatrick BM, Fordyce JA, Gavrilets S (2009) Pattern, process and geographic modes of speciation. Journal of Evolutionary Biology, 22, 2342-2347.

Flot J-F, Tillier A, Samadi S, Tillier S (2006) Phase determination from direct sequencing of length-variable DNA regions. Molecular Ecology Notes, 6, 627-630.

Fluxus Technology Ltd. (2010) Network. Available from: http://www.fluxusengineering.com/sharenet.htm.

Forbes AA, Powell THQ, Stelinski LL, Smith JJ, Feder JL (2009) Sequential sympatric speciation across trophic levels. Science, 323, 776-779.

Francis CM, Borisenko AV, Ivanova NV, et al. (2010) The role of DNA barcodes in understanding and conservation of mammal diversity in Southeast Asia. Plos One, 5, e12575.

Furey NM, Thong VD, Bates PJJ, Csorba G (2009) Description of a new species belonging to the Murina 'suilla-group' (Chiroptera: Vespertilionidae: Murininae) from north Vietnam. Acta Chiropterologica, 11, 225-236.

Galan M, Guivier E, Caraux G, Charbonnel N, Cosson J-F (2010) A 454 multiplex sequencing method for rapid and reliable genotyping of highly polymorphic genes in large-scale studies. BMC Genomics, 11, 296.

Galtier N, Nabholz B, Glemin S, Hurst GDD (2009) Mitochondrial DNA as a marker of molecular diversity: a reappraisal. Molecular Ecology, 18, 4541-4550.

Garrick RC, Sunnucks P, Dyer RJ (2010) Nuclear gene phylogeography using PHASE: dealing with unresolved genotypes, lost alleles, and systematic bias in parameter estimation. BMC Evolutionary Biology, 10, 118.

Gavrilets S, Waxman D (2002) Sympatric speciation by sexual conflict. Proceedings of the National Academy of Sciences of the United States of America, 99, 10533-10538.

Geraldes A, Ferrand N, Nachman MW (2006) Contrasting patterns of introgression at X-linked loci across the hybrid zone between subspecies of the European rabbit (Oryctolagus cuniculus). Genetics, 173, 919-933.

Guillot G (2008) Inference of structure in subdivided populations at low levels of genetic differentiation-the correlated allele frequencies model revisited. Bioinformatics, 24, 22222228.

Hasegawa M, Kishino H, Yano TA (1985) Dating of the human-ape splitting by a molecular clock of mitochondrial DNA. Journal of Molecular Evolution, 22, 160-174.

Heled J, Drummond AJ (2010) Bayesian inference of species trees from multilocus data. Molecular Biology and Evolution, 27, 570-580.

Hey J (2006) Recent advances in assessing gene flow between diverging populations and species. Current Opinion in Genetics \& Development, 16, 592-596.

Hey J (2010a) The divergence of chimpanzee species and subspecies as revealed in multipopulation isolation-with-migration analyses. Molecular Biology and Evolution, 27, 921-933.

Hey J (2010b) Isolation with migration models for more than two populations. Molecular Biology and Evolution, 27, 905-920.

Hey J (2011) Documentation for IMa2. Available from: https://bio.cst.temple.edu/ hey/program_files/IMa2/Using_IMa2_8_24_2011.pdf. 
Hey J, Nielsen R (2004) Multilocus methods for estimating population sizes, migration rates and divergence time, with applications to the divergence of Drosophila pseudoobscura and D. persimilis. Genetics, 167, 747-760.

Hey J, Nielsen R (2007) Integration within the Felsenstein equation for improved Markov chain Monte Carlo methods in population genetics. Proceedings of the National Academy of Sciences of the United States of America, 104, 2785-2790.

Hudson RR, Kaplan NL (1985) Statistical properties of the number of recombination events in the history of a sample of DNA sequences. Genetics, 111, 147-164.

Hulva P, Fornuskova A, Chudarkova A, et al. (2010) Mechanisms of radiation in a bat group from the genus Pipistrellus inferred by phylogeography, demography and population genetics. Molecular Ecology, 19, 5417-5431.

Husband BC, Sabara HA (2004) Reproductive isolation between autotetraploids and their diploid progenitors in fireweed, Chamerion angustifolium (Onagraceae). New Phytologist, 161, $703-713$.

Jackson DE (2008) Sympatric speciation: perfume preferences of orchid bee lineages. Current Biology, 18, R1092-R1093.

Jakobsson M, Rosenberg NA (2007) CLUMPP: a cluster matching and permutation program for dealing with label switching and multimodality in analysis of population structure. Bioinformatics, 23, 1801-1806.

Jukes TH, Cantor CR (1969) Evolution of protein molecules. In: Mammalian protein metabolism (ed Munro HH), pp. 21-132. Academic Press, New York.

Kingston T, Francis CM, Akbar Z, Kunz TH (2003) Species richness in an insectivorous bat assemblage from Malaysia. Journal of Tropical Ecology, 19, 67-79.

Kingston T, Jones G, Akbar Z, Kunz TH (1999) Echolocation signal design in Kerivoulinae and Murininae (Chiroptera: Vespertilionidae) from Malaysia. Journal of Zoology, 249, 359374.

Kingston T, Rossiter SJ (2004) Harmonic-hopping in Wallacea's bats. Nature, 429, 654-657.

Kisel Y, Barraclough TG (2010) Speciation has a spatial scale that depends on levels of gene flow. American Naturalist, 175, 316-334.

Kumar S, Subramanian S (2002) Mutation rates in mammalian genomes. Proceedings of the National Academy of Sciences of the United States of America, 99, 803-808.

Kuo H-C (2004) Systematics of bats of genus Murina in Taiwan (Chiroptera: Vespertilionidae). MS Thesis, National Taiwan University, Taipei.

Kuo H-C (2013) Phylogeography and diversification of Taiwanese bats. PhD Thesis, Queen Mary, University of London, London.

Kuo H-C, Chen S-F, Fang Y-P, Flanders J, Rossiter SJ (2014) Comparative rangewide phylogeography of four endemic Taiwanese bat species. Molecular Ecology, 23, 35663586.

Kuo H-C, Chen S-F, Rossiter SJ (2013) Development and characterisation of microsatellite loci for Murina gracilis (Vespertilionidae) and cross-species amplification in two other congeneric species. Conservation Genetics Resources, 5, 1117-1120.

Kuo H-C, Fang Y-P, Csorba G, Lee L-L (2009) Three new species of Murina (Chiroptera: Vespertilionidae) from Taiwan. Journal of Mammalogy, 90, 980-991. 
801 Larsen PA, Marchán-Rivadeneira MR, Baker RJ (2010) Natural hybridization generates 802 mammalian lineage with species characteristics. Proceedings of the National Academy of 803

Librado P, Rozas J (2009) DnaSP v5: a software for comprehensive analysis of DNA polymorphism data. Bioinformatics, 25, 1451-1452.

Llopart A, Elwyn S, Lachaise D, Coyne JA (2002) Genetics of a difference in pigmentation between Drosophila yakuba and Drosophila santomea. Evolution, 56, 2262-2277.

Losos JB, Glor RE (2003) Phylogenetic comparative methods and the geography of speciation. Trends in Ecology \& Evolution, 18, 220-227.

Lue K-Y, Lin S-M (2008) Two new cryptic species of Takydromus (squamata: lacertidae) from taiwan. Herpetologica, 64, 379-395.

Lynch JD (1989) The gauge of speciation: on the frequencies of modes of speciation. In: Speciation and its Consequences (eds Otte D, Endler JA), pp. 527-553. Sinauer Associates, Sunderland, MA.

Mallet J (2005) Hybridization as an invasion of the genome. Trends in Ecology \& Evolution, 20, 229-237.

Mao X, He G, Zhang J, Rossiter SJ, Zhang S (2013) Lineage divergence and historical gene flow in the Chinese horseshoe bat (Rhinolophus sinicus). Plos One, 8, e56786.

Mao X, Zhang J, Zhang S, Rossiter SJ (2010) Historical male-mediated introgression in horseshoe bats revealed by multilocus DNA sequence data. Molecular Ecology, 19, 13521366.

Margulies M, Egholm M, Altman WE, et al. (2005) Genome sequencing in microfabricated highdensity picolitre reactors. Nature, 437, 376-380.

Mattern MY, McLennan DA (2000) Phylogeny and speciation of felids. Cladistics, 16, 232-253.

Melo-Ferreira J, Vilela J, Fonseca MM, et al. (2014) The elusive nature of adaptive mitochondrial DNA evolution of an arctic lineage prone to frequent introgression. Genome Biology and Evolution, 6, 886-896.

Muir G, Schlötterer C (2005) Evidence for shared ancestral polymorphism rather than recurrent gene flow at microsatellite loci differentiating two hybridizing oaks (Quercus spp.). Molecular Ecology, 14, 549-561.

Nadachowska K, Babik W (2009) Divergence in the face of gene flow: the case of two newts (Amphibia: Salamandridae). Molecular Biology and Evolution, 26, 829-841.

Nesi N, Nakoune E, Cruaud C, Hassanin A (2011) DNA barcoding of African fruit bats (Mammalia, Pteropodidae). The mitochondrial genome does not provide a reliable discrimination between Epomophorus gambianus and Micropteropus pusillus. Comptes Rendus Biologies, 334, 544-554.

Nielsen R, Wakeley J (2001) Distinguishing migration from isolation: A Markov chain Monte Carlo approach. Genetics, 158, 885-896.

Papadopulos AST, Baker WJ, Crayn D, et al. (2011) Speciation with gene flow on Lord Howe Island. Proceedings of the National Academy of Sciences of the United States of America, 108, 13188-13193.

Petit RJ, Excoffier L (2009) Gene flow and species delimitation. Trends in Ecology \& Evolution, 24, 386-393. 
844 Phillimore AB, Orme CDL, Thomas GH, et al. (2008) Sympatric speciation in birds is rare:

Polzin T, Daneshmand SV (2003) On Steiner trees and minimum spanning trees in hypergraphs. Operations Research Letters, 31, 12-20.

Posada D (2008) jModelTest: Phylogenetic model averaging. Molecular Biology and Evolution, 25, 1253-1256.

Pritchard JK, Stephens M, Donnelly P (2000) Inference of population structure using multilocus genotype data. Genetics, 155, 945-959.

Rambaut A, Drummond AJ (2007) Tracer v1.4. Available from http://tree.bio.ed.ac.uk/software/tracer/.

Randi E (2008) Detecting hybridization between wild species and their domesticated relatives. Molecular Ecology, 17, 285-293.

Rice WR (1984) Disruptive selection on habitat preference and the evolution of reproductive isolation - a simulation study. Evolution, 38, 1251-1260.

Rosenberg NA (2004) DISTRUCT: a program for the graphical display of population structure. Molecular Ecology Notes, 4, 137-138.

Rossiter SJ, Benda P, Dietz C, Zhang S, Jones G (2007) Rangewide phylogeography in the greater horseshoe bat inferred from microsatellites: implications for population history, taxonomy and conservation. Molecular Ecology, 16, 4699-4714.

Schmieder DA, Kingston T, Hashim R, Siemers BM (2010) Breaking the trade-off: rainforest bats maximize bandwidth and repetition rate of echolocation calls as they approach prey. Biology Letters, 6, 604-609.

Slatkin M (1982) Pleiotropy and parapatric speciation. Evolution, 36, 263-270.

Stephens M, Scheet P (2005) Accounting for decay of linkage disequilibrium in haplotype inference and missing data imputation. American Journal of Human Genetics, 76, 449462.

Stephens M, Smith NJ, Donnelly P (2001) A new statistical method for haplotype reconstruction from population data. American Journal of Human Genetics, 68, 978-989.

Strasburg JL, Rieseberg LH (2011) Interpreting the estimated timing of migration events between hybridizing species. Molecular Ecology, 20, 2353-2366.

Struebig MJ, Kingston T, Petit EJ, et al. (2011) Parallel declines in species and genetic diversity in tropical forest fragments. Ecology Letters, 14, 582-590.

Tamura K, Peterson D, Peterson N, et al. (2011) MEGA5: molecular evolutionary genetics analysis using maximum likelihood, evolutionary distance, and maximum parsimony methods. Molecular Biology and Evolution, 28, 2731-2739.

Tavaré S (1986) Some probabilistic and statistical problems in the analysis of DNA sequences. In: Some mathematical questions in biology: DNA sequence analysis (ed Miura RM), pp. 5786. American Mathematical Society, Providence, Rhode Island.

Toews DPL, Brelsford A (2012) The biogeography of mitochondrial and nuclear discordance in animals. Molecular Ecology, 21, 3907-3930.

Turelli M, Barton NH, Coyne JA (2001) Theory and speciation. Trends in Ecology \& Evolution, 16, 330-343.

Vallo P, Benda P, Cerveny J, Koubek P (2013) Conflicting mitochondrial and nuclear paraphyly in small-sized West African house bats (Vespertilionidae). Zoologica Scripta, 42, 1-12. 
888

889

890

891

892

893

894

895

896

897

898

899

900

901

902

903

904

905

906

907

908

909

910

911

912

913

914

915

916

917

918

\section{Supporting information}

920 Systematic notes on the focal species.

921 Selection of a demographic model for the ncDNA *BEAST analysis.

922 Tests of positive selection.

923 Tests for sex-biased dispersal in Murina gracilis and M. recondita.

924 Figure S1 Median-joining networks built for the Murina gracilis complex based on ten 925 microsatellite flanking regions.

Via S (2001) Sympatric speciation in animals: the ugly duckling grows up. Trends in Ecology \& Evolution, 16, 381-390.

Voris HK (2000) Maps of Pleistocene sea levels in Southeast Asia: shorelines, river systems and time durations. Journal of Biogeography, 27, 1153-1167.

Watterson GA (1975) On the number of segregating sites in genetical models without White MJD (1978) Modes of speciation. W. H. Freeman and Company, San Francisco, CA.

Woerner AE, Cox MP, Hammer MF (2007) Recombination-filtered genomic datasets by information maximization. Bioinformatics, 23, 1851-1853.

Yu HT (1995) Patterns of diversification and genetic population structure of small mammals in Taiwan. Biological Journal of the Linnean Society, 55, 69-89.

Yule GU (1924) A mathematical theory of evolution based on the conclusions of Dr J. C. Willis. Philosophical Transactions of the Royal Society B-Biological Sciences, 213, 21-87.

\section{Data accessibility}

GenBank accessions for new Murina eleryi sequences are KT762291-KT762293 for COI, KT762294-KT762306 for Cyt- $b$ and KT778876-KT779034 (unphased) for microsatellite flanking regions. GenBank accessions for microsatellite flanking sequences of $M$. gracilis and $M$. recondita generated via 454-Pyrosequencing are KT794560-KT795100. For all focal taxa studied, we created an archive comprising (1) alignments of phased sequences at two microsatellite flanking regions used to produce Fig. 2, (2) an alignment of concatenated Cyt- $b$ and COI genes used to produce Fig. 3a, (3) alignments of phased sequences at 10 microsatellite flanking regions used to produce Fig. $3 \mathrm{~b}$ and (4) input data for the four-taxon IM analyses. This archive is accessible with the Dryad entry: XXX.

\section{Authors' contributions}

H.-C.K., S.-F.C., Y.-P.F, C.-H. Chen and C.-H. Chou collected tissue samples from Taiwanese taxa; G.C., B.K.L. and J.L.E. provided tissue samples of M. eleryi; H.-C.K. conducted laboratory work under the supervision of SJR, and performed statistical analyses with input from S.J.R, J.A.C. and J.D.P. H.-C.K. wrote the paper with S.J.R and all authors participated in discussions. 
926 Figure S2 Marginal densities for demographic parameters from a four-taxon IM analysis of the 927 Murina gracilis complex.

928 Figure S3 Marginal densities for demographic parameters from IM analyses for the six two929 taxon pairs of the Murina gracilis complex.

930 Figure S4 A mitochondrial gene tree used in tests of positive selection.

931 Figure S5 Samples of Murina gracilis and M. recondita analysed for sex-biased dispersal.

932 Figure S6 Corrleograms of individual-level analyses of sex-biased dispersal in Murina gracilis 933 and $M$. recondita.

934 Table S1 External measurements showing body sizes of $M$. gracilis and its relatives.

935 Table S2 Details of 13 voucher specimens of Murina eleryi sampled for genetic analyses.

936 Table S3 Primer pairs for amplification of flanking regions of ten microsatellite loci.

937 Table S4 Estimated proportions of 'foreign' genetic composition for four individual bats of 938 Murina recondita as inferred from STRUCTURE analyses.

939 Table S5 IM estimates based on all four taxa from the Murina gracilis complex.

940 Table S6 IM estimates for six pairs of two taxa from the Murina gracilis complex.

941 Table S7 Sources of mitochondrial sequences used in maximum likelihood analyses in PAML to 942 test for signatures of positive selection.

943 Table S8 Predictions and results of the population-level tests - based on four statistics - for 944 evidence of male-biased dispersal in M. gracilis and M. recondita. 




14 loci

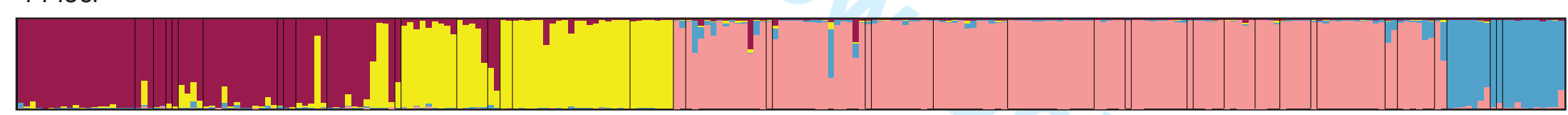

13 loci: purge A9

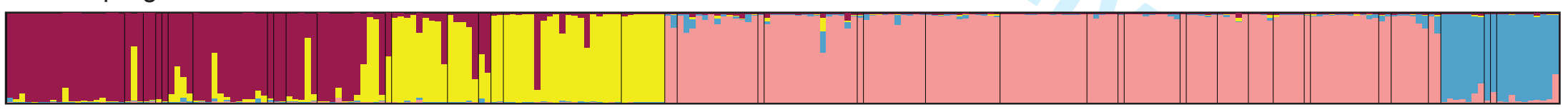

13 loci: purge A122

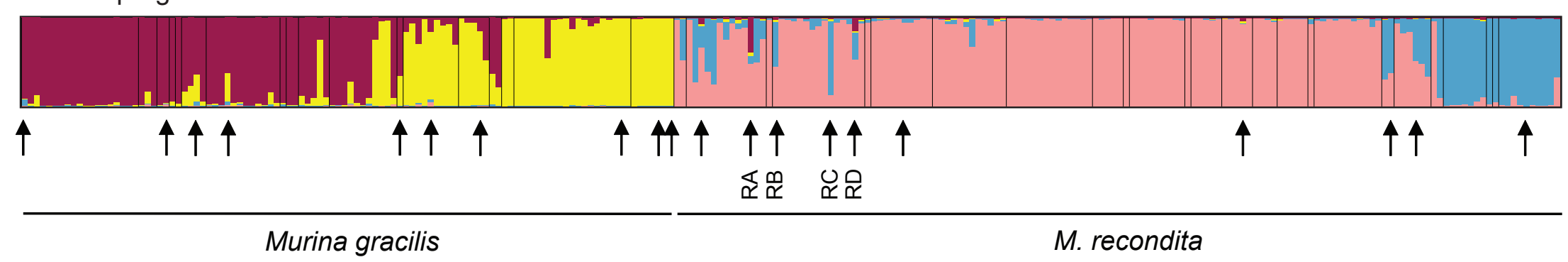


Figure 2

A9 (121 bp)
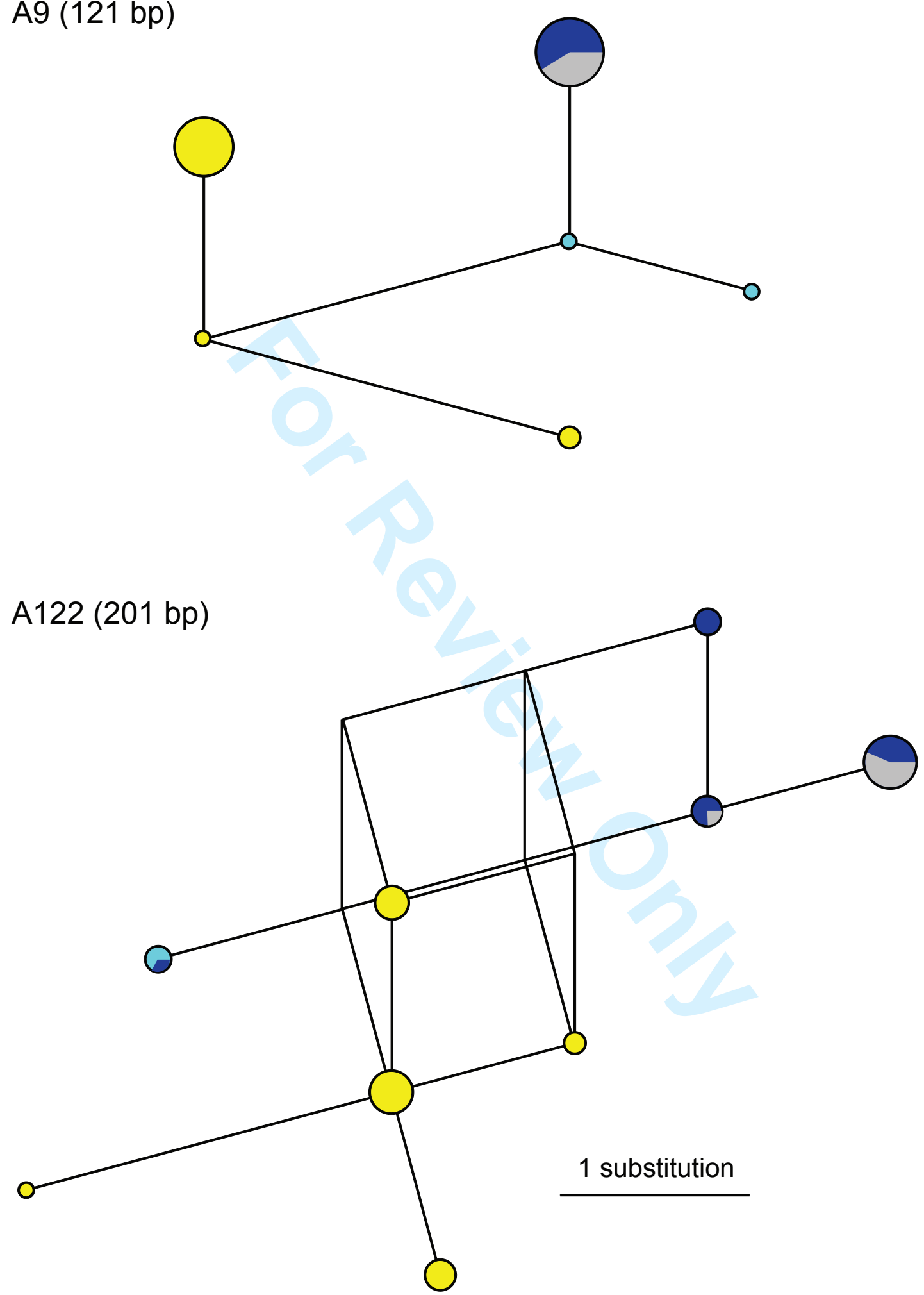


\section{Figure 3}

(a)
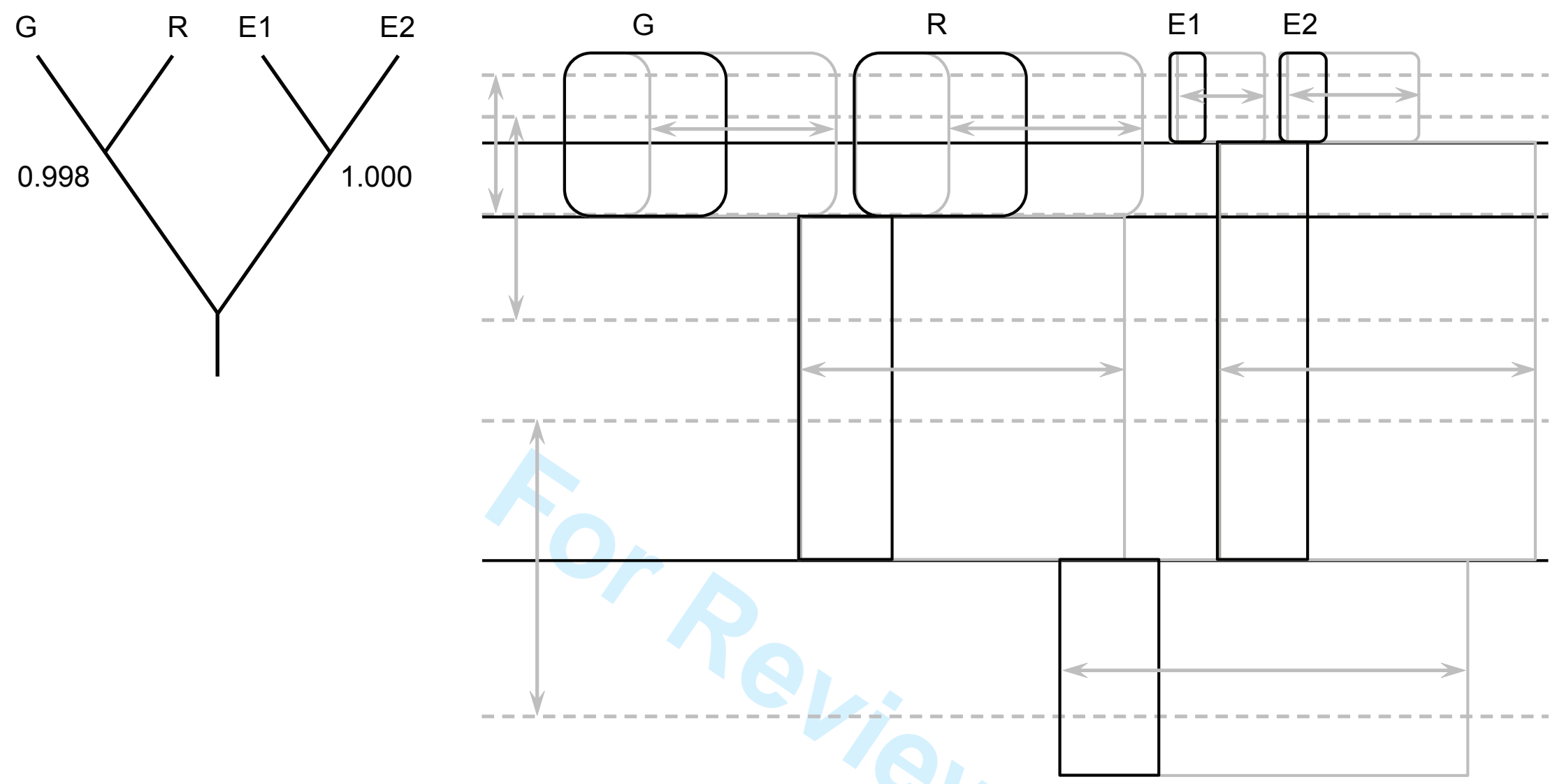

(b)

Figure 4

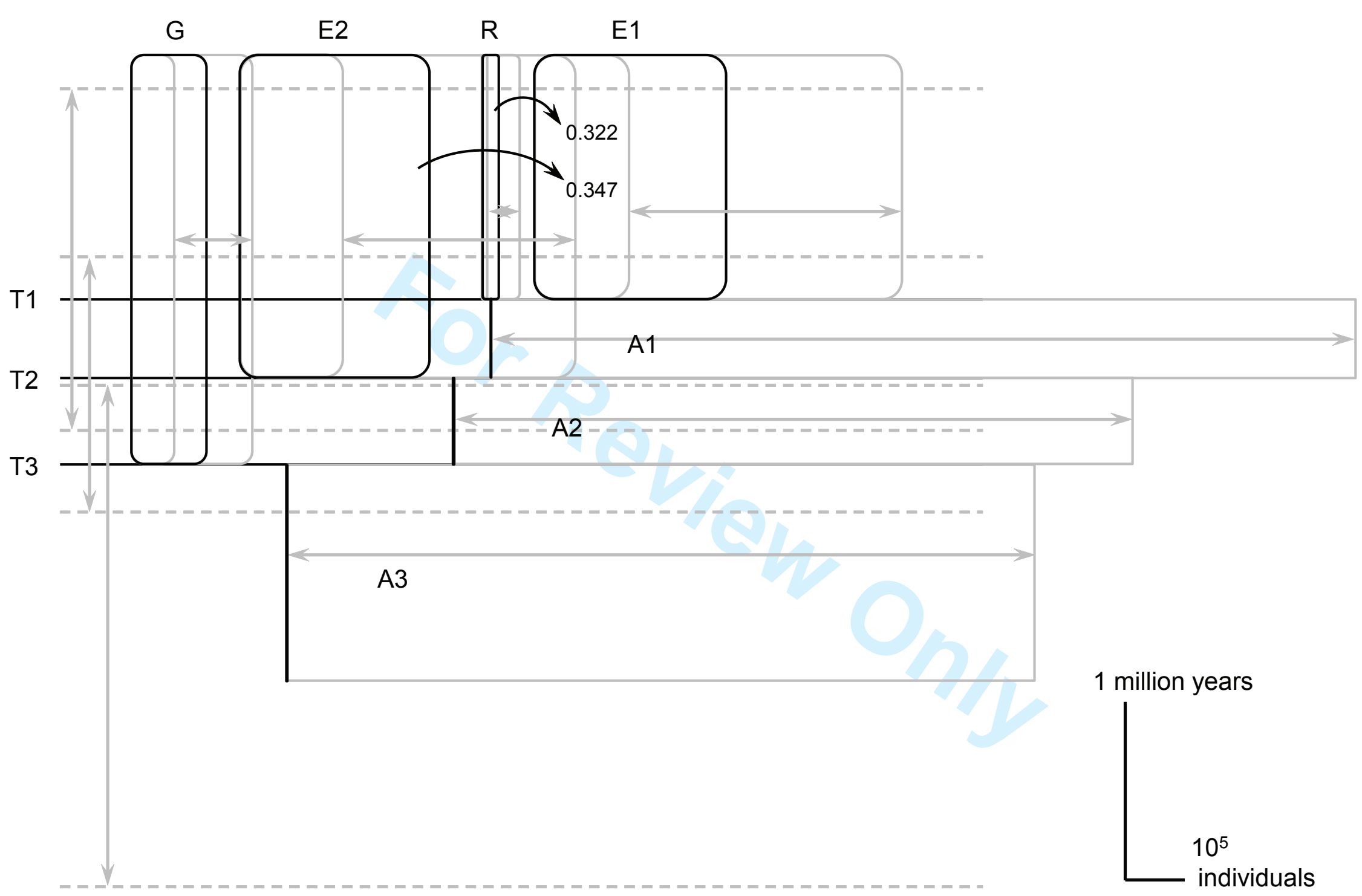




\section{Figure 5}
